CLINICAL STUDY

\title{
Leptin, free leptin index, insulin resistance and liver fibrosis in children with non-alcoholic fatty liver disease
}

\author{
Valerio Nobili ${ }^{1}$, Melania Manco ${ }^{1}$, Paolo Ciampalini ${ }^{2}$, Vincenzo Diciommo ${ }^{3}$, Rita Devito ${ }^{4}$, Fiorella Piemonte ${ }^{5}$, \\ Donatella Comparcola ${ }^{1}$, Roberto Guidi ${ }^{1}$ and Matilde Marcellini ${ }^{1}$ \\ ${ }^{1}$ Liver Unit, ${ }^{2}$ Endocrinolgy Department, ${ }^{3}$ Epidemiology Unit, ${ }^{4}$ Pathology Department and ${ }^{5}$ Molecular Medicine department, Research Institute, 'Bambino \\ Gesù' Children's Hospital, S Onofrio 4 Square, 00165 Rome, Italy
}

(Correspondence should be addressed to M Manco; Email: melaniamanco@tiscali.it)

\begin{abstract}
Objective: Prevalence of non-alcoholic fatty liver disease (NAFLD) among children is increasing dramatically. It is unclear why some patients develop steatohepatitis (NASH), fibrosis and cirrhosis from steatosis, and others do not. A role for leptin has been claimed. This study aims to evaluate the relationship between leptin, insulin resistance (IR) and NAFLD in children.

Design and methods: In 72 biopsy-proven NAFLD children (aged 9-18 years; 51M/21F), fasting leptin and its soluble receptor (sOB-R) were measured; free leptin index (FLI) was calculated as leptin/sOB-R; IR was estimated by homeostasis model assessment (HOMA-IR) and insulin sensitivity index (ISIcomp); glucose tolerance by oral glucose tolerance test (OGTT). Percentage of total body fat (TBF) by dual-energy X-ray absorptiometry (DXA) was available in 65 patients.

Results: Prevalence of diabetes, impaired fasting and/or after load glucose tolerance was 11\%. HOMA-IR and ISI-comp values were $2.55 \pm 1.39$ and $4.4 \pm 2$. NASH was diagnosed in 38 and simple steatosis in 25 children; diagnosis was indeterminate in 29 children. Increased fibrosis, mostly of mild severity, was observed in 41 patients. Median NAFLD activity (NAS) score was $3.42 \pm 1.60$. According to histology, levels of leptin and FLI increased as steatosis (leptin from $11.9 \pm 6.3$ in score 1 to $17.4 \pm 6.9$ in score 2 $(P=0.01)$ and $22.2 \pm 6.8 \mathrm{ng} / \mathrm{ml}$ in score $3(P<0.001)$; FLI $2.56 \pm 1.40,3.57 \pm 0.34,4.45 \pm 0.64$ respectively $(P=0.05)$ ); ballooning (from $13.7 \pm 6.7$ in score 1 to $17 \pm 7.5$ in score $2(P=0.001)$ and $22.1 \pm 7.1 \mathrm{ng} / \mathrm{ml}$ in score $3(P=0.01)$; FLI $2.81 \pm 1.50,3.40 \pm 1.65,4.57 \pm 1.67(P=0.01$ between 0 and 2)); fibrosis (from $14.3 \pm 7$ to $18.3 \pm 6.9 ; P=0.03$; FLI $3.03 \pm 1.57$ vs $3.92 \pm 077 ; P<0.05$ ) and NAS score (score 1-2: 12.9 \pm 6.9 ; score 3-4: $17 \pm 6.9(P=0.01)$; score 5-7: $22.9 \pm 7.5 \mathrm{ng} / \mathrm{ml}(P=0.03)$; FLI $2.70 \pm 1.53,3.12 \pm 1.53,4.58 \pm 1.57 P=0.01$ and $P=0.05$ between $1-2$ vs $3-4$ and $3-4$ vs $5-7$ respectively) worsened. Higher leptin correlated with more severe steatosis, ballooning and NAS score $\left(r_{0}=0.6,0.4\right.$ and 0.6 respectively; for all $\left.P<0.001\right)$; FLI with ballooning $\left(r_{0}=0.4, P<0.0001\right)$, steatosis $\left(r_{0}=0.5, P<0.0001\right)$ and NAS score $\left(r_{0}=0.5, P<0.0001\right)$.

Conclusions: Leptin and liver injury correlated independently of age, BMI and gender in the present study. Nevertheless, any causative role of leptin in NAFLD progression could be established. Thus, studies are needed to define whether the hormone plays a major role in the disease.
\end{abstract}

European Journal of Endocrinology 155 735-743

\section{Introduction}

Non-alcoholic fatty liver disease (NAFLD) has become one of the most common causes of chronic liver disease in many countries (1). The reported prevalence of hypertransaminasemia and/or liver brightness on ultrasonography varies among children from 10 to $77 \%(2,3)$.

The exact mechanisms leading to hepatic triglyceride accumulation and subsequent hepatocellular damage in NAFLD patients are incompletely understood. Insulin resistance (IR) and hyperinsulinemia are clearly important as elements of a vicious cycle. They act to increase free fatty acid (FFA) flux into the liver and drive hepatic triglyceride production, through increased de novo lipogenesis (4) and inhibited FFA oxidation (4). Conversely, hepatic triglyceride accumulation subsequently leads to hepatic IR by interfering in the insulin response cascade $(5,6)$. Disturbances within the regulatory systems of lipid partitioning result in lipotoxicity and steatosis. However, hepatic lipid accumulation does not universally lead to hepatocellular injury, suggesting that additional secondary insults are important (7). It is unclear why some patients develop simple steatosis, whereas others manifest progression from steatohepatitis (NASH) to fibrosis and cirrhosis.

Leptin, the product of the $o b$ gene, is an adipocytederived hormone that can modulate food intake and 
several metabolic functions (8). In human hepatic liver cells, leptin attenuates some insulin-induced activities causing IR (9, 10). Leptin is also expressed and synthesised by the activated hepatic stellate cells (HSC) (11). The hormone also exerts pro-inflammatory and profibrogenic function in murine liver exposed to hepatotoxic chemicals (12), and up-regulates the expression of proinflammatory and pro-angiogenic cytokines in HSC (13). Together, these observations support a role for leptin in the pathogenesis of human NAFLD. However, to the best of our knowledge, no data are available on levels of the hormone's soluble receptor (sOB-R) in NAFLD. Nevertheless, levels of sOB-R can provide an indication of free leptin; the free leptin index (FLI) being defined as the ratio of leptin to sOB-R (14), which may be a more accurate determinant of leptin function. The leptin receptor belongs to the type 1 cytokine receptor family $(15,16)$ and various mRNA splice variants of this receptor have been identified (16). A soluble form of the leptin receptor lacking the cytoplasmic domain is generated by proteolytic cleavage of the membrane-bound receptor and represents the main leptin-binding activity in human blood (17). Although IR is considered pivotal for the development of fatty liver (18) and increased serum leptin may play an important role, few data have been reported in paediatric populations regarding both $\operatorname{IR}(3,19,20)$ and leptin (19-21). A limit of previous studies (3, 19-21) was that diagnosis has been made taking into account elevated serum aminotransferase levels and echogenic liver pattern, and not liver biopsy, which is regarded as 'gold standard' for NAFLD diagnosis (22).

The present study aims to evaluate serum leptin levels, FLI and prevalence of IR in a case series of children, in which NAFLD has been diagnosed by liver percutaneous biopsy.

\section{Material and methods}

\section{Patients}

Seventy-two untreated patients (51 males and 21 females), aged 9-18 years, admitted to the Liver Unit of the 'Bambino Gesù' Hospital from June 2002 to April 2004, were included in the study. Patients were referred to the Unit since persistently elevated serum aminotransferase levels coupled with a diffusely echogenic liver pattern in imaging studies, suggestive of liver fatty infiltration. The diagnosis of NAFLD was confirmed by percutaneous liver biopsy in all cases. All patients were HCV RNA-PCR negative. The known causes of steatosis including alcohol abuse ( $\geq 140 \mathrm{~g} /$ week), total parenteral nutrition and use of drugs (e.g. valproate, amiodarone or prednisone), were excluded. Hepatitis A, B, C, D, E and G, cytomegalovirus and Epstein-Barr virus infections were ruled out by appropriate tests. Autoimmune liver disease, metabolic liver disease, Wilson's disease and $\alpha$-1-antitrypsin were ruled out using standard clinical and laboratory evaluation as well as liver biopsy features. The body mass index (BMI) was computed as the weight in kilograms divided by the square of the height in metres. BMI $z$ score was calculated to compare BMI whilst taking into account differences in gender and age. The $z$ score represents the number of S.D. above or below the considered population mean value based on standardised tables for children (23). Obesity was defined as a BMI above 2s.D., which corresponds to the 97th adjusted for age and gender. Hypertriglyceridemia and hypercholesterolemia were diagnosed for serum levels of triglycerides and cholesterol higher than those reported as normal for age, gender and race (24). Hypertension was diagnosed according to the guidelines of the Fourth Task Force on blood pressure control in children (25).

The study protocol was conformed to the Declaration of Helsinki and to the recommendations of the Ethics Committee at the Children's Hospital and Research Institute, 'Bambino Gesù'. The nature and the purpose of the study were carefully explained before informed consent was requested from each patient or responsible guardian.

\section{Evaluation of glucose metabolism and insulin sensitivity}

All patients underwent a 2-h oral glucose tolerance test (OGTT) with the standard $1.75 \mathrm{~g}$ of glucose $/ \mathrm{kg}$, or maximum of $75 \mathrm{~g}$. Glucose tolerance was determined according to the American Diabetes Association classification (26).

Values of IR were determined by the homeostatic model assessment (HOMA-IR) using the formula: IR = (insulin $\times$ glucose)/22.5 (27); insulin sensitivity was determined by the insulin sensitivity index (ISI-comp) derived from OGTT using the formula: ISI $=(10$ 000/ square root of (fasting glucose $X$ fasting insulin) $X$ (mean glucose $\times$ mean insulin during OGTT)) (28).

\section{Evaluation of total body fat}

In a subgroup of 65 subjects, total body fat (TBF) was estimated from total body dual-energy X-ray absorptiometry (DXA) scans made with a QDR-1500 total body scanner (Hologic, Inc., Waltmann, MA, USA) in the pencil beam mode with the enhanced whole body analysis (software version 5.67). With this technique, composition is estimated from the attenuation of X-rays pulsed synchronously between 70 and $140 \mathrm{kV}$ with the line frequency for each pixel of the scanned image. A step phantom with six fields of acrylic and aluminium of various thicknesses and known absorptive properties was scanned alongside each subject to serve as an external standard for the analysis of tissue composition. The same physician positioned the subjects, performed the scans and the analysis according to the operator's manual. 


\section{Biochemical assays}

Serum was stored at $-80{ }^{\circ} \mathrm{C}$ for later assays. Serum leptin was assayed by commercial ELISA kit in accordance with the manufacturer's instruction (Lincho Research, St Charles, MO, USA). Intra- and inter-assay coefficients of variation are 4.2 and $4.5 \%$ respectively. The sensitivity of the method is $0.5 \mathrm{ng} / \mathrm{ml}$. Levels of sOB-R were measured using an ELISA commercial kit (Alexis Biochemicals, San Diego, CA, USA), with a limit of sensitivity of $0.4 \mathrm{U} / \mathrm{ml}$ and intraassay coefficient of variation of $6.0-6.3 \%$. FLI was calculated as the ratio of leptin to sOB-R. Plasma glucose was measured in triplicate by the glucose oxidase technique on a Beckman glucose analyzer (Beckman, Fullerton, CA, USA); plasma insulin by a specific RIA (MYRIA Technogenetics, Milan, Italy). Serum triglycerides, and total and high density lipoprotein (HDL) cholesterol were measured spectrophotometrically.

\section{Liver histology}

Biopsies were performed in all children using an automatic core biopsy device (Biopince, Amedic, Kista, Sweden) with an 18-G needle, $150 \mathrm{~mm}$ long and the ability to cut tissue with lengths up to $33 \mathrm{~mm}$ with extreme precision. Liver biopsies were at least $15 \mathrm{~mm}$ long and read by the same liver pathologist, who was unaware of the patient's clinical and laboratory data. Biopsies were routinely processed (formalin-fixed, paraffin-embedded) and analysed in sections stained with: (i) hematoxilin and eosin, for overall assessment of parenchymal architecture, hepatocyte abnormalities and inflammatory infiltrates; (ii) Van Gieson, for assessment of fibrosis and architectural changes; (iii) PAS-D, after diastase predigestion, to highlight debris in portal macrophages and Kupffer cells as well as eosinophilic globules in periportal hepatocytes (characteristic of endoplasmic reticulum storage disease, namely $\alpha-1$ antitrypsin); and (iv) Perl's (Prussian blue stain) to estimate iron storage in hepatocytes and sinusoidal lining cells. Additionally, immunohistochemical staining with $\alpha-1$ antitrypsin was used to exclude $\alpha$ 1 antitrypsin associated liver disease. The main histological features commonly described in NAFLD/NASH including steatosis, inflammation (portal and lobular), hepatocyte ballooning and fibrosis were scored using the scoring system for NAFLD according to the guidelines of the NIH-sponsored NASH Clinical Research Network (29). Briefly, steatosis was graded on a four-point scale: grade 0 , steatosis involving $<5 \%$ of hepatocytes; grade 1 , steatosis involving up to $33 \%$; grade 2, steatosis involving $33-66 \%$ and grade 3 , steatosis involving $>66 \%$. Lobular inflammation was graded on a four-point scale: grade 0 , no foci; grade 1 , less than two foci per $200 \times$ field; grade 2 , two to four foci per $200 \times$ filed; grade 3 , more than four foci per
$200 \times$ field. Hepatocyte ballooning was graded from 0 to 2: 0 , none; 1 , few balloon cells; 2 , many/prominent balloon cells. The stage of a fibrosis was quantified in a four-point scale: stage 0, no fibrosis; stage1, perisinusoidal or periportal; stage 2, perisinusoidal and portal/ periportal; stage 3 , bringing and stage 4 , cirrhosis. Other features, such as zonal distribution of steatosis, presence of microvesicular steatosis, glycogenated nuclei, lipogranulomas, PAS-D cells, acidophil bodies and Mallory bodies also were recorded. Portal tract inflammation was graded from 0 to $3(0$, none; 1 , mild; 2 , moderate; 3 , severe). Features of steatosis (0-3), lobular inflammation (0-3) and hepatocyte ballooning $(0-2)$ were combined in a score ranging from 0 to 8 , named NAFLD activity score (NAS). Cases with NAS $\geq 5$ are diagnostic of NASH and cases with NAS $\leq 2$ are diagnostic of simple steatosis, whereas cases in between are considered indeterminate (29). Iron storage was scored from 0 to 4 according to Searle (30).

\section{Statistical analysis}

Data are presented as mean \pm s.D. or median \pm s.D., as stated, and/or number (proportion) of affected patients. The Kolmogorov-Smirnov goodness-of-fit test was applied for determining whether sample data are likely to derive from a normal-distributed population. Triglycerides, alanine aminotransferase (ALT), aspartate aminotransferase (AST), gamma glutamyl transpeptidase $(\gamma \mathrm{GT})$, FLI and histological grades diverged significantly from normal distribution. The non-parametric Spearman's correlation coefficient, based on ranking the two variables, which makes no assumption about the distribution of the values, was used to assess significant correlations among variables. The parametric Pearson's correlation was calculated only to take into account the effect of obesity and age on IR. MannWhitney $U$ test, one-way ANOVA, Kruskal-Wallis test and Bonferroni's post hoc test for multiple comparisons were carried out by standard techniques, whenever appropriate. The level of significance was set at $\alpha=$ 0.05. SPSS 11.5 for Windows (SPSS Inc., Chicago, IL, USA) was used for statistical analysis.

\section{Results}

Table 1 shows anthropometric and biochemical data of the studied population. Table 2 illustrates mean values \pm s.D. of the main variables in the population divided by sex and age.

\section{Glucose metabolism}

Mean values of HOMA-IR and ISI-comp were 2.55 \pm 1.39 and $4.4 \pm 2$ respectively (Table 1 ). HOMA-IR and ISI-comp correlated with BMI $\left(r_{0}=0.349, P=0.004\right.$ and $r_{0}=-0.223, P=0.075$ respectively) and body 
Table 1 Anthropometric, clinical and biochemical characteristics of the studied population $(n=72)$. Data are presented as mean \pm S.D. and/or number (proportion) of affected patients or median \pm S.D. (triglycerides, aspartate aminotransferase (AST), alanine aminotransferase (ALT), gamma glutamyl transpeptidase $(\gamma \mathrm{GT})$, non-alcoholic fatty liver disease (NAFLD) acitvity score (NAS) and free leptin index (FLI).

\begin{tabular}{|c|c|c|}
\hline & Mean \pm s.D. $\boldsymbol{n}(\%)$ & Range \\
\hline Age (years) & $12.0 \pm 3.2$ & $9-18$ \\
\hline $\mathrm{BMI}\left(\mathrm{kg} / \mathrm{m}^{2}\right)$ & $26.3 \pm 4.1$ & $15.2-38.3$ \\
\hline BMI $z$-score & $1.8 \pm 0.8$ & $0.8-5.0$ \\
\hline $\operatorname{TBF}(\%)^{\mathrm{a}}$ & $25.73 \pm 6.67$ & $5-38.9$ \\
\hline Obesity & $29(40.2 \%)$ & \\
\hline Cholesterol (mg/dl) & $152 \pm 32$ & $75-222$ \\
\hline Triglyceride $(\mathrm{mg} / \mathrm{dl})$ & $86 \pm 44.5$ & $28-348$ \\
\hline Hypercholesterolemia & 24 Patients (33\%) & \\
\hline Hypertriglyceridemia & $39(61 \%)$ & \\
\hline AST (IU/I) & $42 \pm 16$ & $19-90$ \\
\hline ALT (IU/I) & $60 \pm 31$ & $10-192$ \\
\hline$\gamma \mathrm{GT}(\mathrm{IU} / \mathrm{I})$ & $23 \pm 20$ & $10-130$ \\
\hline NAS score & $3.42 \pm 1.60$ & $1-7$ \\
\hline Fasting glucose (mg/dl) & $82 \pm 11$ & $60-138$ \\
\hline Fasting insulin (uU/l) & $12.5 \pm 6.4$ & $3.5-30.7$ \\
\hline HOMA-IR & $2.55 \pm 1.39$ & $0.67-6.29$ \\
\hline ISI-comp & $4.4 \pm 2.0$ & $1.26-9.16$ \\
\hline IGT/IFG & $6(0.083)$ & \\
\hline Type II diabetes & $2(0.03 \%)$ & \\
\hline Serum leptin $(\mathrm{ng} / \mathrm{ml})$ & $16.5 \pm 7.7$ & $3.0-43.0$ \\
\hline $\mathrm{sOB}-\mathrm{R}(\mathrm{ng} / \mathrm{ml})$ & $5.03 \pm 0.49$ & $3.74-6.28$ \\
\hline FLI & $3.34 \pm 1.70$ & $0.77 \pm 8.87$ \\
\hline
\end{tabular}

To convert glucose to millimolar per litre, multiply by 0.05551 . To convert insulin values to picomolar per litre, divide by 7.175 . To convert triglycerides to millimolar per litre, multiply by 0.01129 . To convert cholesterol values to millimolar per litre, multiply by 0.02586 . Leptin values normally range from 10 to $15 \mathrm{ng} / \mathrm{ml}$. BMI, body mass index; HOMA-IR, homeostatic model assessment of insulin resistance; IGT, impaired glucose tolerance; IFG, impaired fasting glucose; ISI-comp, insulin sensitivity index; sOB-R, soluble leptin receptor; TFM, total fat mass.

${ }^{a}$ Data on total body fat (TBF) were available only in 65 children.

weight $\left(r_{0}=0.262, \quad P=0.035\right.$ and $r_{0}=-0.362$; $P=0.003)$.

Sixty-four $(88.8 \%)$ patients were glucose tolerant; two children had previously unrecognised type 2 diabetes (DMT2) and six had impaired glucose metabolism (impaired fasting glucose (IFG), impaired glucose tolerance (IGT) or both). The latter patients were significantly older $(14.2 \pm 2.7$ vs $11.8 \pm 3.2$ years; $P<0.05)$ and insulin resistant, as indicated by a lower ISI-comp (ISI-comp: $3.16 \pm 1.25$ vs $4.57 \pm 2.08$; $P<0.01)$ as compared with glucose tolerant subjects. Conversely, they did not show any significant difference in BMI $z$ score $(1.35 \pm 1.32$ vs $1.94 \pm 0.67 ; P=N S)$ and HOMA-IR failed to discriminate glucose-tolerant and -intolerant patients $(2.95 \pm 1.75$ vs $2.49 \pm 1.34$; $P=N S$ ). Five of them were in pubertal stage III or IV.

\section{Liver histology}

Histological findings are summarised in Table 3. Steatosis was present in all biopsies. It was mostly macrovescicular and, frequently associated with microvescicular lesions. The pattern of steatosis was diffuse or scattered lobular, and, only, in ten cases, it showed zonal distribution. In $59(82 \%)$ biopsies, inflammation was present. The inflammatory infiltrate was mainly composed of lymphocytes and neutrophils and when granulomas were present, mononuclear histiocytic cells and eosinophils were associated. Hepatocyte ballooning was present in $34(47 \%)$ biopsies, whereas apoptotic cells were observed occasionally. PAS-D-positive cells containing phagocytosed-cell debris were present in the portal tract and/or in the sinusoids. Glycogenated nuclei of variable dimension were found in 38 cases (53\%); this nuclear change was noted mostly in zone 1 . No Mallory hyaline was noted in any case and mild iron deposition was present in three cases. According to the NAS, a diagnosis of NASH was formulated in $13(18 \%)$ patients (NAS $\geq 5$ ), a diagnosis of simple steatosis (NAS $\leq 2$ ) was made in $26(36 \%)$ patients, whereas $33(29 \%)$ were considered indeterminate. Increased fibrosis was observed in $41(57 \%)$ patients. It was mostly of mild (stage 1) severity with only four children showing septal

Table 2 Main anthropometric, clinical and biochemical characteristics of the studied population according to gender and age. Data are presented as mean \pm s.D. of affected patients or median \pm s.D. (triglycerides, AST; ALT, NAS score and FLI).

\begin{tabular}{|c|c|c|c|c|c|c|}
\hline & \multicolumn{2}{|c|}{ 7-10 Years } & \multicolumn{2}{|c|}{$11-14$ Years } & \multicolumn{2}{|c|}{ 15-18 Years } \\
\hline & Boys $(n=11)$ & Girls $(n=5)$ & Boys $(n=20)$ & Girls $(n=10)$ & Boys $(n=20)$ & Girls $(n=6)$ \\
\hline BMI $\left(\mathrm{kg} / \mathrm{m}^{2}\right)^{\star}$ & $24.89 \pm 3.20$ & $21.41 \pm 3.29$ & $24.82 \pm 4.38$ & $26.48 \pm 5.13$ & $28.69 \pm 4.65$ & $29.35 \pm 5.02$ \\
\hline BMI $z$ score & $2.34 \pm 0.64$ & $1.99 \pm 0.54$ & $1.46 \pm 0.99$ & $1.66 \pm 0.48$ & $1.73 \pm 0.49$ & $1.65 \pm 0.54$ \\
\hline TBF (\%) & $28.13 \pm 3.51$ & $27.23 \pm 10.22$ & $24.12 \pm 8.58$ & $23.99 \pm 5.22$ & $24.07 \pm 5.96$ & $26.70 \pm 3.98$ \\
\hline HOMA-IR & $1.92+1.04$ & $1.74+1.07$ & $2.09+1.37$ & $2.66+1.35$ & $3.09+1.43$ & $3.51+1.23$ \\
\hline ISI-comp* & $5.74 \pm 2.61$ & $4.90 \pm 2.98$ & $4.99 \pm 2.03$ & $3.27 \pm 0.67$ & $3.61 \pm 1.72$ & $3.13 \pm 1.68$ \\
\hline ALT (IU/I) & $53.63 \pm 31.40$ & $52.33 \pm 28.29$ & $62.25 \pm 18.74$ & $77.60 \pm 24.78$ & $68.21 \pm 40.90$ & $90.56 \pm 55.89$ \\
\hline AST (IU/I) & $47.25 \pm 18.85$ & $37 \pm 7.29$ & $39.63 \pm 11.87$ & $50.70 \pm 37.34$ & $46.79 \pm 21.20$ & $56.89 \pm 33.4$ \\
\hline NAS score & $3.75 \pm 1.98$ & $2.33 \pm 0.58$ & $2.88 \pm 1.37$ & $4.30 \pm 1.41$ & $3.07 \pm 1.49$ & $4.56 \pm 2.01$ \\
\hline Leptin $(\mathrm{ng} / \mathrm{ml})^{\dagger, \ddagger}$ & $15.67 \pm 6.71$ & $9.54 \pm 1.84$ & $13.34 \pm 5.53$ & $22.63 \pm 6.76$ & $16.17 \pm 10.09$ & $23.09 \pm 12.34$ \\
\hline $\mathrm{sOB}-\mathrm{R}(\mathrm{ng} / \mathrm{ml})$ & $5.29 \pm 0.50$ & $5.13 \pm 0.06$ & $5.17 \pm 0.54$ & $4.92 \pm 0.30$ & $4.88 \pm 0.54$ & $5.17 \pm 0.544 .9$ \\
\hline $\mathrm{FLI}^{\dagger, \neq}$ & $3.01 \pm 1.44$ & $1.86 \pm 0.34$ & $2.66 \pm 1.29$ & $4.64 \pm 1.51$ & $3.35 \pm 2.18$ & $4.46 \pm 1.89$ \\
\hline
\end{tabular}

ANOVA analysis showed a significant effect of age *on BMI $(P=0.05)$, ISI-comp $(P=0.04)$ in boys; of age ${ }^{\dagger}$ on NAS score $(P=0.03)$, fasting leptin $(P=0.02)$ and FLI $(P=0.003)$ in girls. ${ }^{\ddagger}$ Significant differences between sexes were found at age 11-14 years in NAS score $(P=0.003)$, leptin $(P=$ $0.02)$, FLI $(P=0.01)$; at $15-18$ years in NAS score $(P=0.04)$. 
Table 3 Histological findings in the patient population $(n=72)$.

\begin{tabular}{lcccc}
\hline $\begin{array}{l}\text { Grade/ } \\
\text { stage }\end{array}$ & Steatosis & $\begin{array}{c}\text { Necroin- } \\
\text { flammation }\end{array}$ & Ballooning & Fibrosis \\
\hline 0 & - & $13(18.0 \%)$ & $37(51.3 \%)$ & $31(43.0 \%)$ \\
1 & $27(37.5 \%)$ & $53(73.6 \%)$ & $19(26.3 \%)$ & $35(48.6 \%)$ \\
2 & $26(36.1 \%)$ & $6(8.3 \%)$ & $11(15.2 \%)$ & $2(2.7 \%)$ \\
3 & $19(26.3 \%)$ & - & $4(5.5 \%)$ & $4(5.5 \%)$ \\
4 & - & - & - & - \\
\hline
\end{tabular}

Number of patients affected and percentage in parentheses. Biopsies were scored according to Kleiner et al. (25).

fibrosis (stage 3). No patient showed cirrhotic stage disease on liver biopsy. Table 3 shows levels of fasting circulating leptin in children with and without liver fibrosis. Children with liver fibrosis were significantly older (14.2 vs 12.4 years; $P=0.001$ ), had significantly higher BMI (27.3 vs $\left.24.7 \mathrm{~kg} / \mathrm{m}^{2} ; P=0.003\right)$, not different levels of serum cholesterol (161.2 vs $147.2 \mathrm{mg} / \mathrm{dl} ; P=\mathrm{NS}$ ), but higher triglycerides (90 vs $67 \mathrm{mg} / \mathrm{dl} ; P=0.003$ ), as compared with those without fibrosis. However, prevalence of dislipidemia, levels of liver enzymes as well as the AST/ALT ratio were not different between patients with and without fibrosis. Among patients with impaired glucose metabolism (DMT2, IGT and/or IFG), 50\% had a NAS score $\geq 5$ and fibrosis was present in up to $75 \%$ of them.

Inflammation significantly correlated with the percent of TBF $\left(r_{0}=0.4, P=0.002\right)$ and BMI $z$ score $\left(r_{0}=0.3, P=0.002\right)$. A statistically significant correlation was also found between fibrosis and inflammation $\left(r_{0}=0.26, P=0.04\right)$.

\section{Leptin}

Levels of serum leptin and FLI were or trended to be significantly different among groups, when the studied population was divided according to the histologic assessment (Table 4; Fig. 1). Highly significant positive correlations (Table 4) were found between leptin and steatosis, ballooning and NASH score; higher levels of leptin correlating with higher histological scores $\left(r_{0}=0.6,0.4\right.$ and 0.6 respectively; for all correlations, $P<0.001)$. Both fibrosis and inflammation showed weaker but statistically significant correlations with leptin ( $r_{0}=0.3 ; P=0.03$ and $0.2, P=0.05$ respectively). FLI correlated with ballooning $\left(r_{0}=0.4, P<0.0001\right)$, steatosis $\left(r_{0}=0.5, P<0.0001\right)$ and NAS score $\left(r_{0}=0.5\right.$, $P<0.0001)$.

Table 4 includes mean values of BMI $z$ score, TBF, levels of sOB-R, fasting insulin and triglycerides according to the histological features.

No significant difference in serum leptin was found when children were divided according to presence or absence of diabetes, IGT and/or IFG $(16.7 \pm 7.6$ vs $17.1 \pm 9.1 \mathrm{ng} / \mathrm{ml} ; P=\mathrm{NS})$ and obesity $(16.4 \pm 8.4 \mathrm{in}$ obese vs $16.9 \pm 6.7 \mathrm{ng} / \mathrm{ml}$ in normal weight children;
$P=$ NS). No significant correlation was found between leptin and HOMA-IR, ISI-comp and BMI $z$ score.

A stepwise regression was run to ascertain predictors of NAS score among several independent variables, which included leptin, levels of sOB-R, FLI, TBF, BMI $z$ score, ISI, HOMA and fasting triglycerides. In a model which explained $50 \%$ of the variance in the NAS score, circulating leptin was the best predictor $(\beta=0.505$; $P<0.0001)$. The best predictors of fibrosis was BMI $z$ score (explained variance $27 \%, P=0.04$ ) among leptin, FLI, triglycerides and TBF as independent variables. ISI, HOMA-IR and levels of sOB-R were excluded from the model.

\section{Discussion}

The main result of the present study is that increased leptin levels correlate with the degree of liver lesion in NAFLD children. We found that values of fasting leptin are consistently higher than those reported for normal weight healthy children $(31,32)$; and remarkably, hyperleptinemia is independent of age, body weight and IR. We observe that serum leptin increases as steatosis, inflammation, ballooning, fibrosis worsen, reaching concentrations significantly higher in grades 2 and 3 of steatosis, grade 2 of ballooning, fibrosis and for a NAS score higher than 3 (Table 3). So far, in the literature, no study has found such suggestive correlation between leptin levels and liver damage. In adult cohorts, previously published human studies obtained conflicting outcomes (33-37). Chalasani et al. (30) found no differences in levels of fasting leptin between NASH/NAFLD overweight and obese adult subjects. Conversely, serum leptin levels were significantly higher in subjects with NASH as compared with control subjects in a couple of clinical trials $(33,36)$. In mostly lean young military recruits, the increase in serum leptin was out of proportion to BMI (36); whilst in a cohort of overweight to obese subjects, the relation between leptin and NASH was not anymore persistent after adjusting for age, gender and BMI (38). In a different series, serum leptin was increased and the increment associated with the degree of hepatic steatosis (33). To our knowledge, few data are available in childhood $(3,20)$. A couple of previous studies $(3,20)$ found that leptin increased three- to four-fold in NAFLD children as well as in obese control subjects, and with no relation to the BMI (20). In both the studies, liver biopsy was not performed. Diagnosis was formulated according to increased serum aminotransferase levels and echogenic liver pattern. The strength of the present study is that liver biopsy has been performed in all subjects. It is worth noting that, in our series, only the $40 \%$ of children are obese, thus suggesting that it is unfeasible to explain serum leptin elevations and IR only in terms of obesity. Several factors may induce increased levels of leptin, for instance, hyperinsulinemia 
Table 4 Values of leptin, sOB-R, FLI, fasting insulin, triglycerides, HOMA-IR, ISI, BMI $z$ score and TBF by histological grading. Data are represented as mean + s.D. and $P$ values are reported for the Bonferroni's post hoc test for multiple comparisons in variables normally distributed. Data are represented as median + S.D. and $P$ values reported for the KruskalWallis test in skewed variables (triglycerides and FLI).

\begin{tabular}{|c|c|c|c|c|c|c|c|c|c|c|c|}
\hline & Score & $n$ & $\begin{array}{l}\text { Leptin } \\
\text { (ng/ml) }\end{array}$ & $\begin{array}{l}\text { sOB-R } \\
\text { (ng/ml) }\end{array}$ & FLI & $\begin{array}{l}\text { Fasting } \\
\text { insulin } \\
(\mu \mathrm{Ul} / \mathrm{ml})\end{array}$ & $\begin{array}{l}\text { Triglycerides } \\
\text { (mg/dl) }\end{array}$ & HOMA-IR & ISI-comp & BMI $z$ score & TBF $(\%)^{\mathrm{a}}$ \\
\hline \multirow[t]{3}{*}{ Steatosis ${ }^{*, \dagger}$} & 1 & 27 & $11.9 \pm 6.3$ & $5.05 \pm 0.46$ & $2.56 \pm 1.40$ & $11.94 \pm 6.01$ & $90 \pm 33.46$ & $2.81 \pm 1.28$ & $3.92 \pm 1.94$ & $1.67 \pm 0.84$ & $24.5 \pm 7.29$ \\
\hline & 2 & 26 & $17.4 \pm 6.9$ & $5.03 \pm 0.60$ & $3.57 \pm 0.34$ & $10.21 \pm 5.43$ & $78.5 \pm 66.48$ & $2.35 \pm 1.30$ & $4.80 \pm 1.88$ & $1.90 \pm 0.45$ & $26.20 \pm 6.03$ \\
\hline & 3 & 19 & $22.2 \pm 6.8$ & $4.97 \pm 0.39$ & $4.45 \pm 0.64$ & $13.04 \pm 5.76$ & $87 \pm 66.48$ & & $4.69 \pm 1.96$ & $1.96 \pm 0.93$ & $26.51 \pm 6.40$ \\
\hline \multirow[t]{3}{*}{ Inflammation } & 0 & 13 & $13 \pm 7.1$ & $4.91 \pm 0.55$ & $3.30 \pm 2.04$ & $10.40 \pm 6.22$ & $78 \pm 66.30$ & $2.80 \pm 1.74$ & $3.71 \pm 1.71$ & $1.33 \pm 0.99$ & $22.85 \pm 9.28$ \\
\hline & 1 & 53 & $17.2 \pm 7.9$ & $5.04 \pm 0.59$ & $3.39 \pm 1.71$ & $12.10 \pm 5.91$ & $87 \pm 29.07$ & $2.66 \pm 1.23$ & $4.47 \pm 1.99$ & $1.87 \pm 0.50$ & $25.79 \pm 5.94$ \\
\hline & 2 & 6 & $18.9 \pm 6.5$ & $5.14 \pm 0.39$ & $3.19 \pm 0.30$ & $9.85 \pm 2.75$ & $82.5 \pm 51.77$ & $1.93 \pm 0.53$ & $5.75 \pm 1.26$ & $2.60 \pm 1.32$ & $30.50 \pm 2.38$ \\
\hline \multirow[t]{3}{*}{ Ballooning ${ }^{\star, \dagger}$} & 0 & 37 & $13.7 \pm 6.7$ & $5.09 \pm 0.58$ & $2.81 \pm 1.50$ & $11.43 \pm 6.55$ & $78 \pm 110$ & $2.80 \pm 1.54$ & $4.31 \pm 1.89$ & $1.71 \pm 0.75$ & $25.84 \pm 7.30$ \\
\hline & 1 & 17 & $17 \pm 7.5$ & $4.93 \pm 0.38$ & $3.40 \pm 1.65$ & $11.26 \pm 4.93$ & $91 \pm 29.42$ & $2.49 \pm 0.97$ & $4.58 \pm 2.29$ & $1.89 \pm 0.41$ & $25.18 \pm 4.65$ \\
\hline & 2 & 18 & $22.1 \pm 7.1$ & $4.99 \pm 0.36$ & $4.57 \pm 1.67$ & $12.27 \pm 4.97$ & $88 \pm 45.08$ & $2.38 \pm 1.01$ & $4.57 \pm 1.79$ & $2.03 \pm 0.97$ & $25.99 \pm 7.01$ \\
\hline \multirow[t]{2}{*}{ Fibrosis $^{\star, \dagger, \ddagger}$} & 0 & 31 & $14.3 \pm 7$ & $4.96 \pm 0.55$ & $3.03 \pm 1.57$ & $12.07 \pm 6.92$ & $67 \pm 56.66$ & $2.97 \pm 1.50$ & $4.17 \pm 1.99$ & $1.62 \pm 0.77$ & $24.20 \pm 6.75$ \\
\hline & 1 & 41 & $18.3 \pm 7.9$ & $5.04 \pm 0.43$ & $3.92 \pm 0.77$ & $11.36 \pm 4.98$ & $90 \pm 55.60$ & $2.40 \pm 0.96$ & $4.59 \pm 1.93$ & $1.93 \pm 0.50$ & $26.51 \pm 6.39$ \\
\hline \multirow{3}{*}{$\begin{array}{l}\text { NAS } \\
\text { score }^{\star, t, \S}\end{array}$} & $1-2$ & 26 & $12.9 \pm 6.9$ & $5.02 \pm 0.60$ & $2.70 \pm 1.53$ & $11.72 \pm 6.44$ & $73.5 \pm 28.41$ & $2.97 \pm 1.50$ & $3.97 \pm 1.85$ & $1.61 \pm 0.84$ & $24.63 \pm 7.22$ \\
\hline & $3-4$ & 33 & $17 \pm$ & 5.08 & $3.12 \pm 1.53$ & $11.55 \pm 5.96$ & $89 \pm 32.11$ & $2.52 \pm 1.23$ & $4.45 \pm 1.89$ & $1.85 \pm 0.37$ & $26.78 \pm 6.17$ \\
\hline & & 13 & 2 & 70 & 57 & $11.52 \pm 4.96$ & 84.04 & $2.32 \pm 1.01$ & $=2.04$ & \pm 0.9 & \pm 6.60 \\
\hline
\end{tabular}

${ }^{*} P$ values for leptin. $P=0.01$ between steatosis stages 1 and $2 ; P<0.001$ between stage 2 vs $3 . P<0.001$ and $P=0.01$ between ballooning score 0 vs 1 and 1 vs 2 respectively. $P=0.03$ in fibrosis. $P<0.001$ and $P=0.03$ between NAS score $1-2$ vs $3-4$ and $3-4$ vs 5-7 respectively. ${ }^{\top} P$ values for FLI in steatosis $P=0.05$ between 1 and $2 ; P<0.0001$ between 1 and 3 ; in ballooning $P=0.01$ between 0 and 2 ; in fibrosis $P<$ $0.05 ; P=0.01$ and $P=0.05$ bew $N A S$ s Data 


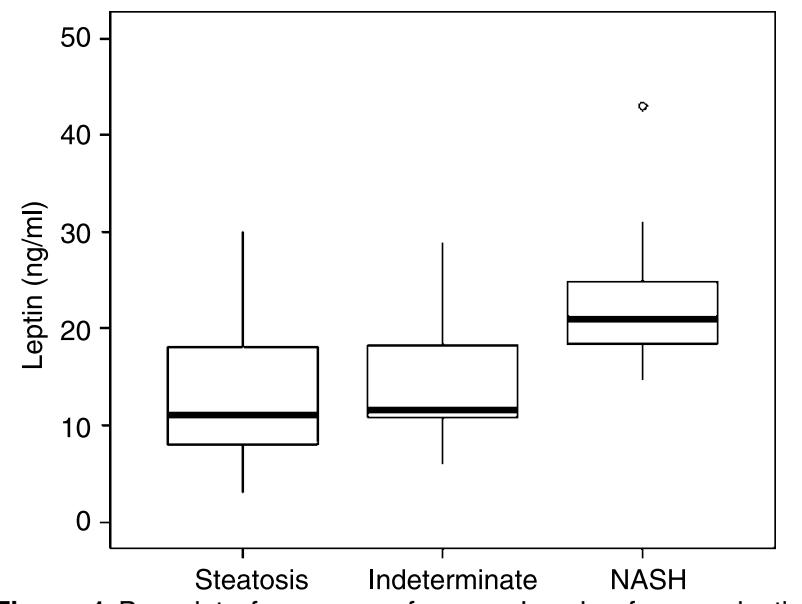

Figure 1 Box plots for groups of cases. Levels of serum leptin (median \pm inter-quartile range) are represented according to the non-alcoholic fatty liver disease (NAFLD) activity (NAS) score. Outliners are shown as circle. $P<0.001$ between simple steatosis and indeterminate, and $P=0.03$ between indeterminate NAFLD. $\mathrm{NASH}$, non-alcoholic steatohepatitis.

or decreased adiponectin (39) as well as a reduced clearance of the hormone. We speculate that hyperleptinemia develops in the early NAFLD before the occurrence of obesity and co-morbidities, promoting IR, overweight and obesity. In response to a dietary chronic FFA overflow to the liver, circulating leptin increases to prevent hepatic lipotoxicity $(33,40)$, as suggested by experimental evidences. A 120-min leptin infusion is able to induce partitioning of TG into oxidative pathways in the liver of lean mice (41) by increasing hepatic acetyl-CoA carboxylase phosphorylation and FFA oxidation. The homeostatic lipid partitioning system may become allostatic, when the FFA overload is persistent (42). The sustained effort to struggle lipotoxicity through increased leptin levels may ultimately result in a status of leptin resistance, with hyperleptinemia not correlating anymore with the degree of liver involvement in the adult age. Therefore, the liver becomes refractory to the anti-steatotic effects of leptin, a state of hepatic leptin resistance that accompanies hepatic IR rather than correcting it (33, 43). Leptin may contribute to hepatic steatosis by promoting IR through reduced insulin signalling in hepatocytes (44). In a later stage, hyperleptinemia may cause hepatic steatosis to turn into steatohepatitis by amplifying selected pro-inflammatory responses (45) up to the development of fibrosis, since it is expressed and synthesised by activated HSC (12), it is able to augment inflammatory and fibrogenic responses in the murine liver exposed to hepatotoxic chemicals (13). The hormone can act in concert with other pro-inflammatory cytokines (45) and pro-fibrotic molecules including IGF-1 (39). To measure them would add valuable information to the present study. In our series, data on IGF-1 and growth hormone were available in 35 consecutive patients (data not shown). Values were within the normal range for both and no relationship was found with liver histology.

Recently, we have also found that older age and higher BMI significant and independent of other risk factors increased the chances of having liver fibrosis in children (46). Every one year increase in age was associated with a 1.3-fold increased risk of developing fibrosis. Similarly, the presence of obesity was associated with a 3.0-fold increased risk as compared with nonobese children (20).

Few studies have examined body composition and hormonal determinants of sOB-R and FLI in adolescents; none, the relation among FLI, sOB-R and NAFLD. We did not find any significant correlation among indexes of body fatness (BMI $z$ score and TBF) and FLI or sOB-R. In addition, we also did not find any relation between levels of fasting insulin and sOB-R in our cohort. These findings suggest a different regulatory pathway for leptin secretion and leptin-binding capacity in adolescent children prone to develop NAFLD. An inverse relationship between BMI and sOB-R has been reported in children $(47,48)$, with both BMI and fat mass contributing significantly to levels of FLI and leptin in anorectic and normal weight adolescent girls, and BMI weakly predicting levels of sOB-bound leptin (48). It has been found that the leptin-binding capacity varies physiologically in relation to increasing adiposity (49), but several dietary and hormonal factors may also determine leptin concentrations in the steady state (47-50).

As far as the role of IR in NAFLD is concerned, in our series, children are mostly insulin-resistant or prone to be, and dislipidemic, but just the $40 \%$ of them are obese. These results confirm previous reports $(3,20)$. We estimated the resistance or, on the other hand, the sensitivity to insulin by using two methods, the HOMAIR and the Matsuda's index, which strongly correlate with the euglycemic hyperinsulinemic clamp $(27,28)$, in order to avoid underestimating the prevalence of IR. Compared with the HOMA-IR, the ISI index represents a composite of both hepatic and peripheral tissue sensitivity to insulin, considering the fasting glucose and insulin values as well as the mean OGTT values of both parameters. In our series, IR and ISI-comp weakly correlate with BMI and body weight, but they did not with leptin or NAFLD score. Kawasaki et al. (19) found a high correlation between hyperinsulinemia and hypertransaminasemia in a group of obese pre-pubertal Japanese children. According to our report, in an adult cohort, Chalasani did not find any relationship between serum leptin, fasting insulin and IR (34). The weakness of the correlation between IR/sensitivity, and BMI or body weight may have a number of explanations. Increased levels of circulating triglycerides and FFAs are able to induce IR independent of weight and BMI as well as intra-muscle fat depots (51). During puberty, changes in sex hormones and the $\mathrm{GH}$ axis can affect, to a certain extent, the insulin sensitivity. 
Moreover, body weight and BMI per se can only approximately reflect body composition and fat distribution, which strongly affect insulin sensitivity.

In conclusion, we find a relationship between leptin levels, FLI and steatosis, ballooning, fibrosis and NAS score in a cohort of children affected by NAFLD with a BMI ranging from normal weight to obesity. However, since a cause-effect relationship cannot be established in the present study, further studies at a molecular level are needed to define to which extent leptin leads to the NAFLD development and progression.

\section{References}

1 Bedogni G, Miglioli L, Masutti F, Tiribelli C, Marchesini G \& Bellentani S. Prevalence of and risk factors for nonalcoholic fatty liver disease: the Dionysos nutrition and liver study. Hepatology 200542 44-52.

2 Wieckowska A \& Feldstein AE. Non-alcoholic fatty liver disease in the paediatric population: a review. Current Opinion in Pediatrics 200517 636-641.

3 Mandato C, Lucariello S, Licenziati MR, Franzese A, Spagnuolo MI, Ficarella R, Pacilio M, Amitrano M, Capuano G, Meli R \& Vajro P. Metabolic, hormonal, oxidative, and inflammatory factors in pediatric obesity-related liver disease. Journal of Pediatrics 2005 $14762-66$

4 Browning JD \& Horton JD. Molecular mediators of hepatic steatosis and liver injury. Journal of Clinical Investigation 2004 114 147-152.

5 Samuel VT, Liu ZX, Qu X, Elder BD, Bilz S, Befroy D, Romanelli AJ \& Shulman GI. Mechanism of hepatic insulin resistance in nonalcoholic fatty liver disease. Journal of Biological Chemistry 2004 279 32345-32353.

6 Schattenberg JM, Wang Y, Singh R, Rigoli RM \& Czaja MJ. Hepatocyte CYP2E1 overexpression and steatohepatitis lead to impaired hepatic insulin signaling. Journal of Biological Chemistry 2005280 9887-9894.

7 Day CP \& James OF. Steatohepatitis: a tale of two 'hits'? Gastroenterology $1998114842-825$.

8 Janeckova R. The role of leptin in human physiology and pathophysiology. Physiological Research 200150 443-459.

9 Cohen B, Novick D \& Rubinstein M. Modulation of insulin activities by leptin. Science $1996 \mathbf{2 7 4} 1185-1188$.

10 Muller G, Ertl J, Gerl M \& Preibish G. Leptin impairs metabolic actions of insulin in isolated rat adipocytes. Journal of Biological Chemistry 1997272 10585-10593.

11 Potter JJ, Womack L, Mezey E \& Anania FA. Transdifferentiation of rat hepatic stellate cells results in leptin expression. Biochemical and Biophysical Research Communications 1998244 178-182.

12 Ikejima K, Honda H, Yoshikawa M, Hirose M, Kitamura T, Takei Y \& Sato N. Leptin augments inflammatory and profibrogenic responses in the murine liver induced by hepatotoxic chemicals. Hepatology 200134 288-297.

13 Aleffi S, Petrai I, Bertolani C, Parola M, Colombatto S, Novo E, Vizzutti F, Anania FA, Milani S, Rombouts K, Laffi G, Pinzani M \& Marra F. Upregulation of proinflammatory and proangiogenic cytokines by leptin in human hepatic stellate cells. Hepatology 200542 1339-1348.

14 Yannakoulia M, Yiannakouris N, Bluher S, Matalas A-L, Klimis-Zacas D \& Mantzoros CS. Body fat mass and macronutrient intake in relation to circulating soluble leptin receptor, free leptin index, adiponectin, and resistin concentrations in healthy humans. Journal of Clinical Endocrinology and Metabolism 200388 1730-1736.

15 Tartaglia L, Dembski M, Weng X, Deng N, Culpepper J, Devos R, Richards G, Campfield L, Clark F, Deeds J, Muir C, Sanker S,
Moriarty A, Moore K, Smutko J, Mays G, Woolf E, Monroe C \& Tepper R. Identification and expression cloning of a leptin receptor, OB-R. Cell 199583 1263-1271.

16 White D \& Tartaglia L. Evidence for ligand-independent homooligomerization of leptin receptor (OB-R) isoforms: a proposed mechanism permitting productive long-form signaling in the presence of excess short-form expression. Journal of Cellular Biochemistry 199973 278-288.

17 Lammert A, Kiess W, Bottner A, Glasow A \& Kratzsch J. Soluble leptin receptor represents the main leptin binding activity in human blood. Biochemical and Biophysical Research Communications 2001283 982-988.

18 Chitturi S \& George J. Interaction of iron, insulin resistance, and nonalcoholic steatohepatitis. Current Gastroenterology Reports $2003518-25$.

19 Kawasaki T, Hashimoto N, Kikuchi T, Takahashi H \& Uchiyama M. The relationship between fatty liver and hyperinsulinemia in obese Japanese children. Journal of Pediatric Gastroenterology and Nutrition 199724 317-321.

20 Schwimmer JR, Deutsch R, Rauch JB, Behling C, Newbury R \& Lavine JE. Obesity, insulin resistance, and other clinicopathological correlates of pediatric nonalcoholic fatty liver disease. Journal of Pediatrics 2003143 500-505.

21 Iorio R, Sepe A, Giannattasio A, Cirillo F, Spagnuolo MI, Franzese A, Fontana S, Aufiero D, Perna F, Vegnente A \& Matarese G. Immune phenotype and serum leptin in children with obesity-related liver disease. Journal of Clinical Endocrinology and Metabolism 200691 341-344.

22 Angulo P. Non-alcoholic fatty liver disease. New England Journal of Medicine 2002346 1221-1231.

23 Cole TJ, Bellizzi MC,, Flegal KM \& Dietz WH. Establishing a standard definition for child overweight and obesity worldwide: international survey. BMJ 2000320 1-6.

24 Brotons C, Ribera A, Perich RM, Abrodos D, Magana P,, Pablo S, Terradas D, Fernandez F \& Permanyer G. Worldwide distribution of blood lipids and lipoproteins in childhood and adolescence: a review study. Atherosclerosis $19981391-9$.

25 National High Blood Pressure Education Program Working Group on High Blood Pressure in Children and Adolescents. The fourth report on the diagnosis, evaluation, and treatment of high blood pressure in children and adolescents. Pediatrics $2004114555-576$.

26 Report of the Expert Committee on the Diagnosis and Classification of Diabetes Mellitus. Diabetes Care 199720 1183-1197.

27 Matthews DR, Hosker JP, Rudenski AS, Naylor BA, Treacher DF \& Turner RC. Homeostasis model assessment: insulin resistance and beta-cell function from fasting plasma glucose and insulin concentrations in man. Diabetologia 198528 412-419.

28 Matsuda M \& De Fronzo RA. Insulin sensitivity indices obtained from oral glucose tolerance testing: comparison with the euglycemic insulin clamp. Diabetes Care 199922 1462-1470.

29 Kleiner DE, Brunt EM, Van Natta M, Behling C, Contos MJ, Cummings OW, Ferrell LD, Liu YC, Torbenson MS, Unalp-Arida A, Yeh M, McCullough AJ \& Sanyal AJ. Nonalcoholic steatohepatitis clinical research network. Design and validation of a histological scoring system for nonalcoholic fatty liver disease. Hepatology 200541 1313-1321.

30 Searle J. Kerr JRF \& Halliday JW. Iron storage disease. In Pathology of the Liver, 2nd edn, Eds RNM MacSwwen, PP Anthony \& PJ Scheuer, Edinburgh: Churchill Livingstone, 1987.

31 Garcia-Mayor RV, Andrade MA, Rios M, Lage M, Dieguez C \& Casanueva FF. Serum leptin levels in normal children: relationship to age, gender, body mass index, pituitary-gonadal hormones, and pubertal stage. Journal of Clinical Endocrinology and Metabolism 199782 2849-2855.

32 Blum WF, Englaro P, Hanitsch S, Juul A, Hertel NT, Muller J, Skakkebaek NE, Heiman ML, Birkett M, Attanasio AM, Kiess W \& Rascher W. Plasma leptin levels in healthy children and adolescents: dependence on body mass index, body fat mass, gender, pubertal stage, and testosterone. Journal of Clinical Endocrinology and Metabolism 199782 2904-2910. 
33 Chitturi S, Farrell G, Frost L, Kriketos A, Lin R, Fung C, Liddle C, Samarasinghe D \& George J. Serum leptin in NASH correlates with hepatic steatosis but not fibrosis: a manifestation of lipotoxicity? Hepatology 200236 403-409.

34 Chalasani N, Crabb DW, Cummings OW, Kwo PY, Asghar A, Pandya PK \& Considine RV. Does leptin play a role in the pathogenesis of human nonalcoholic steatohepatitis? American Journal of Gastroenterology 200398 2771-2776.

35 Nakao K, Nakata K, Ohtsubo N, Maeda M, Moriuchi T, Ichikawa T, Hamasaki K, Kato Y, Eguchi K, Yukawa K \& Ishii N. Association between nonalcoholic fatty liver, markers of obesity, and serum leptin level in young adults. American Journal of Gastroenterology 200297 1796-1801.

36 Uygun A, Kadayifci A, Yesilova Z, Erdil A, Yaman H, Saka M, Deveci MS, Bagci S, Gulsen M, Karaeren N \& Dagalp K. Serum leptin levels in patients with nonalcoholic steatohepatitis. American Journal of Gastroenterology 200095 3584-3589.

37 Giannini E, Botta F, Cataldi A, Tenconi GL, Ceppa P, Barreca T \& Testa R. Leptin levels in nonalcoholic steatohepatitis and chronic hepatitis C. Hepatogastroenterology 199946 242-245.

38 Angulo P, Alba LM, Petrovic LM, Adams LA, Lindor KD \& Jensen MD. Leptin, insulin resistance, and liver fibrosis in human non-alcoholic fatty liver disease. Journal of Hepatology $2004 \mathbf{4 1}$ 943-949.

39 Novosyadlyy R, Tron K, Dudas J, Ramadori G \& Scharf JG. Expression and regulation of the insulin-like growth factor axis components in rat liver myofibroblasts. Journal of Cellular Physiology 2004199 388-398.

40 Lee Y, Wang MY, Kakuma T, Wang ZW, Babcock E, McCorkle K, Higa M, Zhou YT \& Unger RH. Liporegulation in diet-induced obesity. The antisteatotic role of hyperleptinemia. Journal of Biological Chemistry 2001276 5629-5635.
41 Huang W, Dedousis N, Bandi A, Lopaschuk GD \& O’doherty RM. Liver triglyceride secretion and lipid oxidative metabolism are rapidly altered by leptin in vivo. Endocrinology 2006 147 1480-1487.

42 Pickup JC \& Crook MA. Is type II diabetes mellitus a disease of the innate immune system? Diabetologia 199841 1241-1248.

43 Larter CZ \& Farrell GC. Insulin resistance, adiponectin, cytokines in NASH: which is the best target to treat? Journal of Hepatology $200644253-261$.

44 Kaplan LM. Leptin, obesity, and liver disease. Gastroenterology $1998115997-1001$.

45 Day CP. From fat to inflammation. Gastroenterology 2006130 207-210.

46 Nobili V, Marcellini M, Devito R, Ciampalini P, Piemonte F \& Comparcola D. Nonalcoholic fatty liver disease in children: a prospective clinical-pathological study and effect of lifestyle advice. Hepatology $2006 \mathbf{4 4}$ 458-465.

47 Kratzsch J, Lammert A, Bottner A, Seidel G, Mueller J, Thiery J, Hebebrand J \& Kiess W. Circulating soluble leptin receptor and free leptin index during childhood, puberty and adolescence. Journal of Clinical Endocrinology and Metabolism 200287 4587-4594.

48 Landt M. Leptin binding and binding capacity in serum. Clinical Chemistry 200046 379-384.

49 Mantzoros CS. The role of leptin in human obesity and disease: a review of current evidence. Annals of Internal Medicine 1999130 671-680.

50 Manco M, Calvani M \& Mingrone G. Effects of dietary fatty acids on insulin sensitivity and secretion. Diabetes, Obesity and Metabolism $20046402-413$.

Received 26 June 2006

Accepted 31 August 2006 